\title{
A little less conjugation, a little more accuracy
}

\author{
The modification of proteins with fluorophores, drugs and polymers is required for many applications, \\ yet conjugation reactions often generate a heterogeneous mixture of products. A collection of articles in \\ this issue focuses on methods to modify proteins in a site-selective manner.
}

Proteins can fold into an incredibly diverse range of structures despite being made from only a limited number of building blocks the twenty-or-so proteinogenic amino acids. The modular nature of proteins has enabled their evolution into separate species that perform a variety of biological roles; however, it also means that the number of different functional groups present is relatively small. Furthermore, these groups are typically found at many different locations in a protein structure, making it difficult for chemists wishing to selectively modify a specific site. Chemoselective reactions that target a particular functional group - such as the primary amine of a lysine side-chain or the thiol of a cysteine residue - will typically proceed at all of the accessible reactive sites on a protein and can lead to a mixture of products that often possess different properties. When a homogenous conjugate is required, a different synthetic strategy is needed - one that offers precise control over the site at which modification occurs.

A variety of different methods have been developed to provide site-selectivity, such as introducing surface-exposed cysteines ${ }^{1,2}$ as well as harnessing the selectivity of enzymatic reactions ${ }^{3,4}$. Alternatively $\mathrm{N}$-terminal amino acids can sometimes be selectively targeted ${ }^{5}$. Another powerful method involves incorporating unnatural amino acids into proteins through genetic-encoding strategies $^{6-9}$. This enables bio-orthogonal functional groups such as azides, alkynes, ketones, alkenes, tetrazines and norbornenes to be included at specific side-chain positions. Such functional groups are not found elsewhere in proteins and so can be selectively targeted, although it should be noted that genetic incorporation of such amino acids is technically challenging and can significantly reduce the amount of protein produced.

In an Article in this issue Bradley Pentelute and co-workers (page 120) take a different approach, one based on creating a unique micro-environment around the side-chain of a naturally occurring amino acid. They report the discovery of a tetrapeptide - which they call a $\pi$-clamp - that contains a cysteine residue flanked by amino acids that make the thiol of the cysteine more reactive. This thiol can undergo an aromatic substitution reaction with a perfluoroaryl partner. Crucially, however, the perfluoroaryl compound does not react with other cysteines or disulfide bonds. Therefore, incorporating this tetrapeptide into a protein or longer peptide sequence enables the polypeptide chain to be modified selectively. A variety of chemical cargos (such as drugs or fluorophores) can then be covalently attached to the protein by appending them to a perfluoroaryl group.

Pentelute and co-workers demonstrate the versatility of their approach by showing the reaction can occur with the $\pi$-clamp incorporated at the $\mathrm{N}$-terminus, C-terminus or in the middle of the peptide. As proof of the site-selectivity they also use a $\pi$-clamp to selectively modify an antibody that contains 32 native cysteine residues. In the accompanying News \& Views article (page 101), Yichao Huang and Lei Liu highlight that the relatively small size of the $\pi$-clamp tetrapeptide should enable it to be incorporated into a protein with minimal disruption to the protein's structure and properties. They also say it is "quite unexpected" that only four amino acid residues are required to form a unique microenvironment containing a reactive thiol.

As noted earlier, a range of different approaches exist for covalently labelling proteins at specific sites and two Perspective articles in this issue review recent developments in this area. In one, Vijay Chudasama, Antoine Maruani and Stephen Caddick discuss advances in controlling the conjugation site when making antibody-drug conjugates (ADCs) (page 114). Synthetic approaches that use non-selective reactions generate a heterogeneous mixture of ADCs that differ in both the amount of drug loaded on to the antibody as well as the positions at which they are attached. The distinct species that are formed can have very different pharmacokinetics and so tailoring the synthesis to produce the particular conjugates that have more desirable properties offers one opportunity to optimize their efficacy and potentially reduce any side-effects. The Perspective article looks at different conjugation technologies and suitable reaction targets for site-selective reactions, focusing specifically on engineering cysteines, enzyme-directed methods, incorporating unnatural amino acids, targeting reduced disulfides, and selectively targeting glycans.

The other Perspective article (page 103), by Gonçalo Bernardes and co-workers, reviews methods for the site-selective installation of post-translational modifications (PTMs) onto proteins, with a view to their eventual application. Unravelling the biological role of PTMs requires a homogenous protein sample, which is often difficult to isolate from natural sources. As such, Bernardes and colleagues consider the progress that has been made in the fabrication of different PTMs. They also look at the labelling of proteins with fluorescent dyes to enable monitoring of their intracellular trafficking or for measuring the association of two proteins by Förster resonance energy transfer. Another point of discussion concerns which modification strategies are suitable for use inside live cells. The conjugation of proteins with synthetic polymers such as polyethyleneglycol or small molecules can greatly affect the stability, efficacy and immunogenicity of the resulting materials and so the production of ADCs and the modulation of the properties of protein therapeutics are discussed in this context.

The authors of both Perspective articles agree that currently there is not one single approach for site-selective modification that out-performs all others. Rather, different technologies are suited to different applications, different proteins and different synthetic labels. It may not be possible to predict with any certainty how this field will progress (although each Perspective article ends with some thoughts about its future and what challenges remain), nevertheless, it is clear that developing new, site-selective reactions that are easy to implement will help with the rational design and synthesis of new and useful bioconjugates.

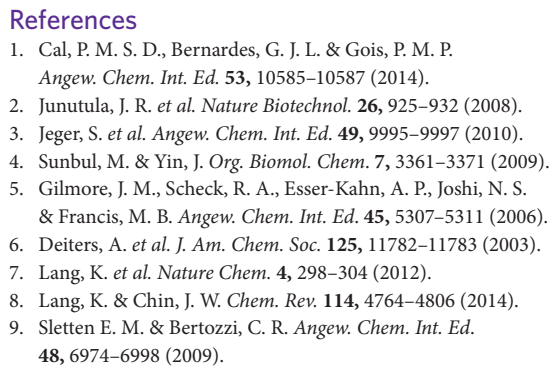

